\title{
Abordaje multifactorial del síndrome de QT largo adquirido en la COVID-19
}

\author{
Juan Mora-Delgado' ${ }^{\text {, Ana Del-Río-Lechuga }}{ }^{2}$, Cristina Lojo-Cruz ${ }^{3}$ \\ 'Servicio de Medicina Interna. Hospital Universitario de Jerez de la Frontera. Jerez de la Frontera (Cádiz). España \\ ${ }^{2}$ Servicio de Cardiología. Hospital Universitario de Jerez de la Frontera. Jerez de la Frontera (Cádiz). España \\ ${ }^{3}$ Servicio de Enfermedades Infecciosas y Microbiología Clínica. Hospital Universitario de Jerez de la Frontera. Jerez de la Frontera (Cádiz). España
}

Recibido: 18/06/2020

Aceptado: $24 / 07 / 2020$

En línea: 31/08/2020

Citar como: Mora-Delgado J, Del-Río-Lechuga A, Lojo-Cruz C. Abordaje multifactorial del síndrome de QT largo adquirido en la COVID-19. Rev Esp Casos Clin Med Intern (RECCMI). 2020 (Ago); 5(2): 93-95. doi: 10.32818/reccmi.a5n2a13.

Cite this as: Mora-Delgado J, Del-Río-Lechuga A, Lojo-Cruz C. Multifactorial approach to long QT syndrome acquired in COVID-19. Rev Esp Casos Clin Med Intern (RECCMI). 2020 (Ago); 5(2): 93-95. doi: 10.32818/reccmi.a5n2a13.

Autor para correspondencia: Juan Mora-Delgado juanmorainternista@gmail.com

\section{Palabras clave}

$\triangleright$ Síndrome de QT largo

$\triangleright$ COVID-19

\begin{tabular}{l} 
Keywords \\
$\triangleright$ Long QT syndrome \\
$\triangleright$ COVID-19 \\
\hline
\end{tabular}

\section{Resumen}

La prolongación del intervalo QT es una medida en ocasiones infravalorada que puede favorecer situaciones pro-arritmogénicas fatales. Muchos pacientes con COVID-19 se están tratando con fármacos que potencialmente prolongan el intervalo QT. Este caso clínico pretende demostrar que la prevención de su aparición y el estudio de otras alteraciones concomitantes que lo favorecen son imprescindibles en el abordaje integral de estos enfermos.

Abstract
The prolongation of the QT interval is a finding that is sometimes undervalued, but that can potentially lead to
the development of arrhythmogenic processes. Many patients are being treated for COVID-19 with drugs that
potentially prolong QT. This clinical case aims to demonstrate that the prevention of its appearance and the stu-
dy of other concomitant alterations that favor it are essential in the comprehensive approach to these patients.

\section{Puntos destacados}

$\triangleright$ La historia de uso de fármacos y otros factores modificables son relevantes: existen diversos eventos laboratoriales que potencian los riesgos de medicamentos para el tratamiento de la COVID-19.

$\triangleright$ Se necesita un protocolo de seguimiento electrocardiográfico durante todo el tratamiento farmacológico.

$\triangleright$ El riesgo-beneficio del tratamiento y las posibles situaciones pro-arritmogénicas fatales se tienen que valorar periódicamente.

\section{Introducción}

Desde que se informó del primer caso a finales de 2019 no se ha demostrado una terapia efectiva frente al coronavirus 2 del síndrome respiratorio agudo grave (SARS-CoV-2) y su enfermedad pulmonar asociada (COVID-19)'. En el contexto de la actual pandemia de COVID-19, existe un gran interés en el uso de cloroquina o hidroxicloroquina en combinación con azitromicina para mejorar la eficacia virucida contra el SARS-CoV-2. Esto contrasta con la preocupación de un mayor riesgo de prolongación de QT y el desarrollo de taquicardia ventricular polimorfa (torsades de pointes)², principalmente en relación con la hidroxicloroquina, que favorece con mayor frecuencia la aparición de bloqueo auriculoventricular respecto a la azitromicina ${ }^{3}$. En general, el intervalo QT corregido (QTC) promedio en personas sanas durante la infancia es de $400 \pm 20$ milisegundos (ms) y aumenta ligeramente después de la pubertad a $420 \pm 20$ ms. Los valores de QTc del percentil 99 son 460 ms (prepubertad), 470 ms en hombres y $480 \mathrm{~ms}$ en mujeres (ambos pospubertad) ${ }^{4}$.

\section{Caso clínico}

Mujer de 78 años que acudió a Urgencias por tos seca, astenia, náuseas y fiebre de hasta $38^{\circ} \mathrm{C}$ de 5 días de evolución. No presentaba alergias medicamentosas conocidas. Sin hábitos tóxicos. Diabetes mellitus tipo 2 diagnosticada hacía 14 años con retinopatía diabética no proliferativa, hipertensión arterial conocida desde hacía 20 años e hipercolesterolemia. Su tratamiento domiciliario era: omeprazol 20 mg, telmisartán 40 mg e hidroclorotiazida 12,5 mg en desayuno, metformina $1 \mathrm{~g}$ y empaglifozina $12,5 \mathrm{mg}$ en desayuno y cena, amlodipino 10 mg e insulina aspart $4 \mathrm{UI}$ en almuerzo, e insulina glargina $16 \mathrm{UI}$ en cena. 
A su llegada al Servicio de Urgencias presentaba estado general regular, normotensa, con febrícula y taquipneica en reposo, precisando oxigenoterapia mediante mascarilla con reservorio. Se auscultaron tonos rítmicos a unos 100 latidos por minuto con hipoventilación global, roncus dispersos y sibilantes aislados.

Se le realizó una analítica en la que destacaba elevación de la proteína C reactiva $(75,6 \mathrm{mg} / \mathrm{l})$ y lactato deshidrogenasa, así como anemia microcítica, linfopenia y elevación del dímero-D (5.339 ng/ml). También se realizó PCR para SARS-CoV-2 (2019), que fue positiva, y se descartó infección por virus de la inmunodeficiencia humana, hepatitis B y $C$.

La radiografía de tórax mostró focos de condensación de predominio periféricos en campos medios e inferiores derechos, así como otro de menor tamaño y densidad más tenue en campo medio izquierdo (Figura 1).

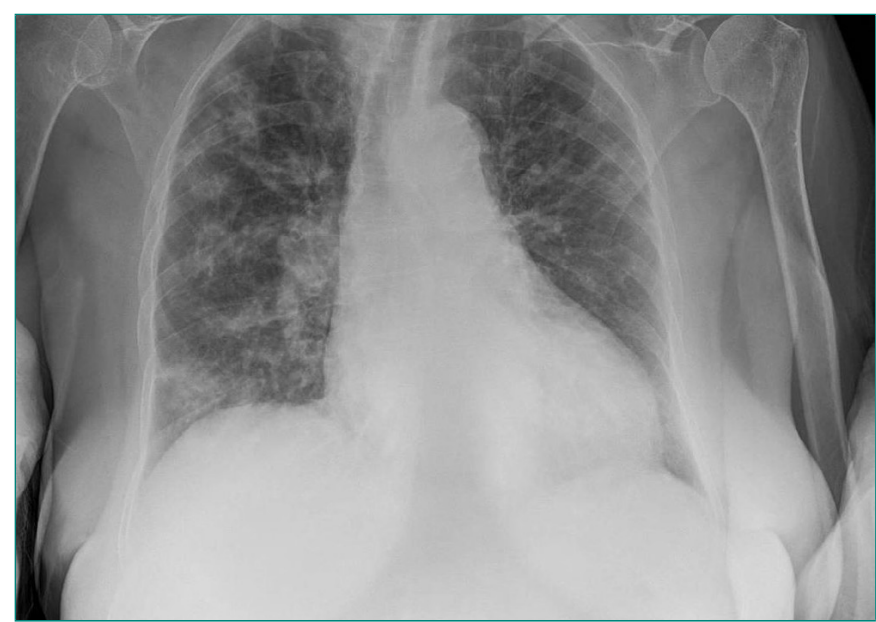

Figura 1. Radiografía de tórax al ingreso, con patrón característico de COVID-19

Al ingreso en la Unidad de Enfermedades Infecciosas, se inició tratamiento con hidroxicloroquina (dosis de carga de 400 mg/12 h el primer día, y 4 días de mantenimiento de 200 mg/12 h), lopinavir/ritonavir (400/100 mg/12 h) y azitromicina (500 mg/día).

A las 48 horas desde el inicio del tratamiento dirigido, se objetivó en un electrocardiograma (ECG) de control (Figura 2) un ritmo sinusal a 54 latidos por minuto, eje normal, QRS estrecho (102 ms), sin alteraciones agudas de la repolarización, intervalo PR 152 ms e intervalo QT corregido mediante fórmula de Fridericia de $463 \mathrm{~ms}$

En relación con la prolongación del intervalo QT, se comprobó en un análisis de control hipopotasemia de $2,6 \mathrm{mEq} / \mathrm{l}(3,5-5,1)$ e hipomagnesemia de $1,18 \mathrm{mg} / \mathrm{dl}(1,6-2,6)$. La paciente permanecía con $\mathrm{SatO}_{2} 95 \%$ con gafas nasales a 3 litros, sin palpitaciones ni dolor torácico, comentando disminución de ingesta desde el ingreso por hiporexia, deposiciones blandas y febrícula.

Dados los hallazgos electrocardiográficos y analíticos, se decidió realizar ECG diario, inicio de sueroterapia con aporte de potasio y magnesio, así como su plementación enteral hipercalórica/hiperproteica. Setenta y dos horas más tarde, tras inicio de reposición hidroelectrolítica de potasio y magnesio vía intravenosa y posteriormente oral, así como suplementación enteral hipercalórica e hiperproteica, se evidenció corrección del intervalo QTcF (corregido por fórmula de Fridericia) (Figura 3); ya presentaba ritmo sinusal a 71 latidos por minuto, eje normal, QRS estrecho (98 ms), sin alteraciones agudas de la repolarización, PR 200 ms y QTcF 423 ms. En un análisis paralelo se evidenció corrección de la hipopotasemia $(4,1 \mathrm{mEq} / \mathrm{l})$ así como de la hipomagnesemia $(1,68 \mathrm{mg} / \mathrm{dl})$. Se completaron 5 días de tratamiento con hidroxicloroquina, azi- tromicina y lopinavir/ritonavir

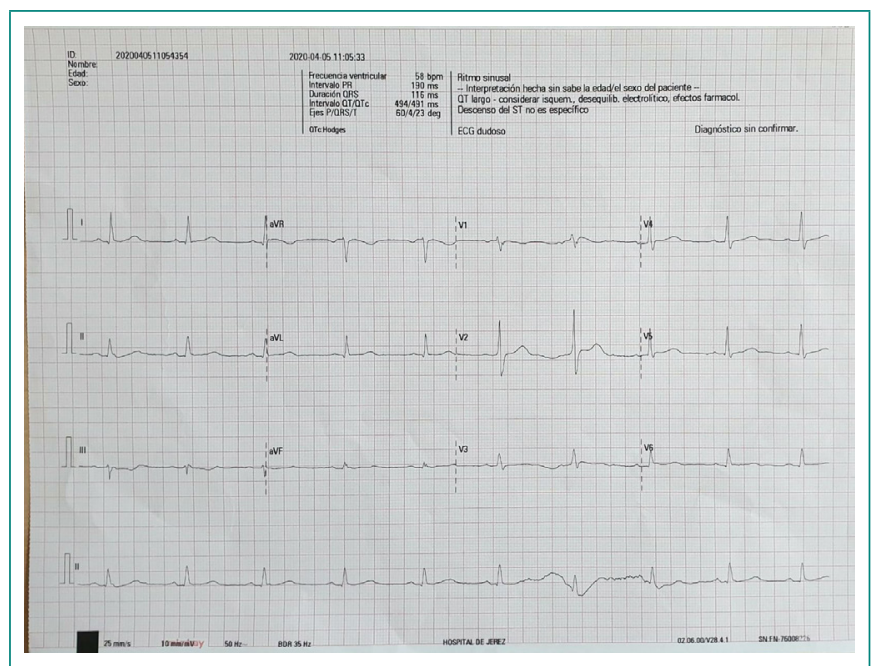

Figura 2. ECG de control a las 48 horas desde el inicio del tratamiento. Intervalo QTCF prolongado

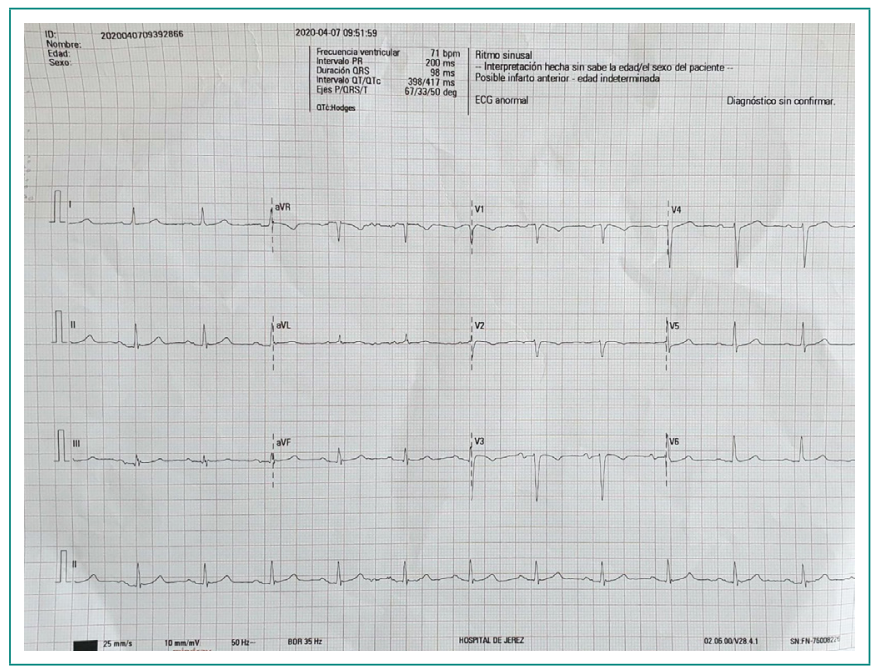

Figura 3. ECG a las 72 horas del inicio de la reposición hidroelectrolítica. Corrección del intervalo QTCF

Se continuaron los controles electrocardiográficos diarios tras finalizar el tratamiento, sin nuevas alteraciones. Se realizó radiografía de tórax de control, con mejoría radiológica evidente. Al duodécimo día de ingreso, clínica y hemodinámicamente estable, fue dada de alta hospitalaria.

\section{Discusión y conclusión}

Varios de los fármacos más utilizados frente a la COVID-19, como la azitromicina y, sobre todo, la hidroxicloroquina, prolongan el intervalo $\mathrm{QT}^{2}$. En el caso de esta última, al ser estructuralmente similar a la quinidina, prolonga el intervalo QT al bloquear la activación del canal de potasio $\mathrm{IKr}^{5}$. Además, tanto la cloroquina como la hidroxicloroquina son metabolizadas por el CYP3A4, y si hay otros medicamentos que inhiben este citocromo podrían elevar sus niveles plasmáticos ${ }^{6}$. Los datos limitados sobre la hidroxicloroquina sugieren que tiene un bajo riesgo de causar torsades de pointes, según su uso habitual en patologías reumatológicas? 
Para el tratamiento de la COVID-19 estos medicamentos se utilizan en pautas de corta duración, otra razón por la que debería ser menor el riesgo de torsades de pointes. Sin embargo, puede haber mayor riesgo de prolongación del intervalo QT en aquéllos con alteraciones hidroelectrolíticas, como fue el caso de nuestra paciente. Aquellos que toman otros medicamentos que prolongan el intervalo QT presentan insuficiencia renal crónica o síndrome de QT largo congénito (Tabla 1).

Hay que obtener el valor basal de QTc del paciente antes de administrar cualquier medicamento cuyo potencial sea prolongar el intervalo QT. La prolongación del intervalo QT en el ECG de 12 derivaciones es un factor de riesgo conocido para la aparición de arritmias ventriculares tanto en jóvenes como en adultos. La prevalencia estimada de esta alteración oscila entre el 8,3-25,8\% en el mundo, siendo la alteración del QT prolongado la que se ha encontrado con mayor frecuencia en diversos estudios de cohorte ${ }^{8}$.

Fisiológicamente, el intervalo QT es la manifestación electrocardiográfica de la despolarización y repolarización ventricular que se produce por el flujo de iones a través de los canales presentes en las membranas de los miocardiocitos.

Existe aún controversia sobre cuál es la mejor fórmula para corregir el intervalo QT. Las cuatro fórmulas más populares son las de Bazzet, Fridericia Framingham y Hodges. En diversos estudios, la fórmula de Bazzet tiende a sobrestimar la prevalencia de QTC largo debido a su modelo lineal. Algunos estudios concluyen que las fórmulas de Fridericia y Framingham son las más consistentes. Además, la fórmula de Fridericia $\left(\mathrm{QTC}=\mathrm{QT} / \mathrm{RR}^{1 / 3}\right)$ presenta mayor precisión en los extremos fisiológicos de la frecuencia cardíaca. Esta última es la que hemos utilizado en nuestro caso clínico, así como de forma general cuando se evaluaron los ECG seriados que se realizaron en nuestros pacientes con COVID-19.

En definitiva, este caso clínico pretende resaltar la importancia de una monitorización adecuada mediante ECG en todo paciente que inicie tratamiento habitual frente a la COVID-19. El control previo al inicio del tratamiento y, posteriormente, cada 24-48 horas, en función de la evolución clínica y electrocardiográfica, sirve para prevenir procesos arritmogénicos que pongan en riesgo la vida del paciente. Si se produce una prolongación del intervalo QT, es esencial no sólo reevaluar el tratamiento dirigido, sino también otras causas independientes sobreañadidas que potencian ese efecto.

\section{Bibliografía}

1. Zhang J, Xie B, Hashimoto K. Current status of potential therapeutic candidates for the COVID-19 crisis. Brain Behav Immun. 2020; 87: 59-73. doi: 10.1016/j. bbi.2020.04.046.

2. Mercuro NJ, Yen CF, Shim DJ, Maher TR, McCoy CM, Zimetbaum PJ, et al. Risk of QT interval prolongation associated with use of hydroxychloroquine with or without concomitant azithromycin among hospitalized patients testing positive for coronavirus disease 2019 (COVID-19). JAMA Cardiol. 2020; e201834. doi: 10.1001/jamacardio.2020.1834.

3. Chorin E, Wadhwani L, Magnani S, et al. QT interval prolongation and torsade de pointes in patients with COVID-19 treated with hydroxychloroquine/azithromycin. Heart Rhythm. 2020; S1547-5271(20)30435-30435. doi: 10.1016/j.hrthm.2020.05.014.

4. Drew BJ, Ackerman MJ, Funk M, Gibler WB, Kligfield P, Menon V, et al; American Heart Association Acute Cardiac Care Committee of the Council on Clinical Cardiology; Council on Cardiovascular Nursing; American College of Cardiology Foundation. Prevention of torsade de pointes in hospital settings: a scientific statement from the American Heart Association and the American College of Cardiology Foundation. J Am Coll Cardiol. 2010; 55(9): 934-947. doi: 10.1016/j.jacc.2010.01.001.

5. Capel RA, Herring N, Kalla M, Yavari A, Mirams GR, Douglas G, et al. Hydroxychloroquine reduces heart rate by modulating the hyperpolarization-activated current If: Novel electrophysiological insights and therapeutic potential. Heart Rhythm. 2015; 12(10): 2186-2194. doi: 10.1016/j.hrthm.2015.05.027.

6. Lee JY, Vinayagamoorthy N, Han K, Kwok SK, Ju JH, Park KS, et al. Association of polymorphisms of cytochrome p450 2d6 with blood hydroxychloroquine levels in patients with systemic lupus erythematosus. Arthritis Rheumatol. 2016 Jan; 68(1): 184-190. doi: 10.1002/art.39402.

7. Chen CY, Wang FL, Lin CC. Chronic hydroxychloroquine use associated with QT prolongation and refractory ventricular arrhythmia. Clin Toxicol (Phila). 2006; 44(2): 173-175. doi:10.1080/15563650500514558.

8. Goldenberg I, Zareba W, Moss AJ. Long QT syndrome. Curr Probl Cardiol. 2008; 33(11): 629-694. doi: 10.1016/j.cpcardiol.2008.07.002

9. Vandenberk B, Vandael E, Robyns T, Vandenberghe J, Garweg C, Foulon V, et al. Which QT correction formulae to use for qt monitoring? J Am Heart Assoc. 2016; 5(6): e003264. doi: 10.1161/JAHA.116.003264.

\begin{tabular}{|c|}
\hline Alteraciones metabólicas \\
\hline Fármacos antiarrítmicos \\
\hline Fármacos antianginosos \\
\hline Fármacos anticolinérgicos \\
\hline Antipalúdicos \\
\hline Azoles \\
\hline Antibióticos \\
\hline Opioides \\
\hline Broncodilatadores \\
\hline Diuréticos \\
\hline Fármacos con acción gastrointestinal \\
\hline Fármacos neurotropos \\
\hline Fármacos psicotropos
\end{tabular}

\begin{tabular}{l}
\hline Hipopotasemia, hipomagnesemia, hipocalcemia, hipotiroidismo, anorexia \\
\hline Quinidina, procainamida, flecainida, propafenona, amiodarona, dronedarona, sotalol \\
\hline Ranolazina, ivabradina \\
\hline Solifenacina, tolterodina \\
\hline Quinidina, quinina, cloroquina, hidroxicloroquina \\
\hline Fluconazol, voriconazol, itraconazol \\
\hline Quinolonas, macrólidos \\
\hline Buprenorfina, metadona \\
\hline$\beta_{2}$ agonistas \\
\hline Tiazídicos, diuréticos del asa \\
\hline Loperamida, ondansetrón, cisaprida, domperidona, metoclopramida \\
\hline Apomorfina, donepezilo, fingolimod \\
\hline $\begin{array}{l}\text { Clorpromacina, haloperidol, amisulpirida, clozapina, olanzapina, quetiapina, risperidona, paliperidona, } \\
\text { citalopram, escitalopram, fluoxetina, trazodona }\end{array}$ \\
\hline
\end{tabular}

Tabla 1. Causas concomitantes más habituales que propician la aparición del síndrome de QT largo en pacientes con COVID-19 